\title{
Magnetic amplification in cylindrical cosmological structure
}

\author{
G. Siemieniec-Oziębło and Z. A. Golda
}

\author{
Astronomical Observatory, Jagellonian University, Faculty of Physics, Astronomy and Applied Computer Science, ul. Orla 171, \\ 30-244 Kraków, Poland
}

Received 29 October 2003 / Accepted 1 April 2004

\begin{abstract}
We derive the amplification of the cosmological magnetic field associated with forming gravitational structure. The self-similar solutions of magnetohydrodynamic equations are computed both in linear and nonlinear regimes. We find that the relatively fast magnetic field enhancement becomes substantial in the nonlinear phase.
\end{abstract}

Key words. cosmology: theory - cosmology: miscellaneous

\section{Introduction}

The hypothesis describing the dynamical role of the primordial magnetic field in the formation and evolution of gravitational structure frequently occurs in the literature (e.g., Wasserman 1978; Kim et al. 1996; Peebles 1995). On the contrary, the inverse trend i.e. the amplification of the magnetic field during density perturbation collapse is not often represented in the context of large-scale structure formation. It is common practice to refer to the constraints for magnetic field amplification set by the density of collapsed matter (e.g., Zeldovich et al. 1980). The hints of magnetic field existence on cosmological scales excites interest not only in the absolute value of a frozen field magnification but also in its growth rate. The early nonlinear and previrial phase of gravitational formation is of particular importance since it results in several megaparsec structures observed as superclusters or filaments. An understanding of the amplification rate of the structure is obviously related to the explanation of the appearance of sufficiently strong frozen-in magnetic fields expected at this stage of collapse.

We investigate the mildly nonlinear collapse of cylindrical gravitational structure and give the growth rate of the primordial magnetic field as a function of the accretion velocity field. The magnetic field growth proceeds intensively during the phase of fluid compression. The magnetic flux for collapsing plasma is conserved and the magnetic strength changes according to the induction equation. It clearly shows that substantial amplification occurs for strongly compressing flows i.e. for growing $\operatorname{div} \boldsymbol{v}-$ analogously to the shock processes. The general expression for $\operatorname{div} v$ is obtained thanks to the self-similar form of the hydrodynamic equation. The self-similar presentation of magnetohydrodynamics becomes possible in the case of rapid density contrast and velocity evolution, when $v$ and

Send offprint requests to: G. Siemieniec-Oziębło, e-mail: grazyna@oa.uj.edu.pl $\delta \propto a^{n}(n>1)$ i.e. when the Lorentz force neglect in the Euler equation is justified.

The plan of the paper is as follows. In Sect. 2 we give the magnetohydrodynamic (MHD) equations for cylindrical structures in comoving coordinates of a flat universe. We also discuss the assumptions and symmetries allowing us to separate the induction equation. In Sect. 3 we derive the self-similar set of hydrodynamic equations. Its linearization and the subsequent comparison with the known velocity solutions are also given. We obtain the general nonlinear relation $\operatorname{div} v$ versus $\delta$. The rate of magnetic field amplification is discussed in Sect. 4 both in the linear and nonlinear regime. In the former we present the analytical expression for amplification and the results of numerical integration in the latter.

\section{Basic equations and perturbation scheme for magnetized fluid}

The magnetic field modifies the standard equations for density perturbations and peculiar velocities. These equations completed by the Faraday equation for the magnetic field have been extensively studied in the context of magnetic field influence on galaxy formation (e.g., Lesch \& Chiba 1995). The linear approximation of the MHD equations has been analyzed in many papers. In the extreme approach, the primordial magnetic field is responsible for the origin of density fluctuations (Wasserman 1978). In his paper the compression of matter is induced by the Lorentz force but the linearized hydrodynamic equations keep both $\rho$ and $\boldsymbol{v}$ as small linear values. There is no fluid back reaction on the magnetic field.

In this paper we apply a different approach. The weak initial and uniform magnetic field $\boldsymbol{B}$ is embedded in the forming large-scale structure which evolves in the dust era. The gravitational collapse changes the strength of the magnetic field in the linear and then nonlinear regime. We are interested in the nonlinear amplification rate of the ordered field. 
The MHD equations are in comoving coordinates given by (e.g., Wassermann 1978)

$$
\begin{aligned}
& \frac{\partial \rho}{\partial t}+3 \frac{\dot{a}}{a} \nabla \cdot \boldsymbol{v}+\frac{1}{a} \nabla \cdot(\rho \boldsymbol{v})=0, \\
& \frac{\partial \boldsymbol{v}}{\partial t}+\frac{\dot{a}}{a} \boldsymbol{v}+\frac{1}{a}(\boldsymbol{v} \cdot \nabla) \boldsymbol{v}=-\frac{1}{a} \nabla \psi-\frac{1}{4 \pi a \rho} \boldsymbol{B} \times(\nabla \times \boldsymbol{B}), \\
& \frac{1}{a^{2}} \Delta \psi=4 \pi G\left(\rho-\rho_{0}\right), \\
& \frac{\partial \boldsymbol{B}}{\partial t}+2 \frac{\dot{a}}{a} \boldsymbol{B}=\frac{1}{a} \nabla \times(\boldsymbol{v} \times \boldsymbol{B}), \\
& \nabla \cdot \boldsymbol{B}=0,
\end{aligned}
$$

where $\rho(t, r)$ is the matter density $\left(\rho_{0}(t)\right.$ means the background density), $\boldsymbol{v}(t, \boldsymbol{r})$ is the peculiar velocity of the matter forming the structure and $\psi(t, r)$ is the density perturbation potential; $a(t)$ is the scale factor given in units $a\left(t_{0}\right)$. For an assumed flat FRW cosmological model we take the initial value of the uniform magnetic field $B_{0}$ at the initial time $t_{0}$.

The temporal evolution analyzed here is restricted to the after recombination phase and we do not discuss the magnetogenesis processes assumed to occur prior to this phase. The small value of the initial magnetic energy density (relative to the matter density) is assumed. Since the field uniformity is maintained within the scale of the forming structure, it does not perturb the background isotropy. The evolving structure and thus the considered field have axial symmetry. The velocity of the accreting fluid is taken for simplicity as $\boldsymbol{v}=\left[v_{r}(t, r), v_{\theta}(t, r), 0\right]$. The assumed cylindrical symmetry is consistent with the isotropy and homogeneity of the FRW model for all length scales large with respect to the coherence scale. Finally, the nonlinear perturbation equations for density fluctuations of presureless matter, velocity field and the magnetic field $\boldsymbol{B}$ are given by

$$
\begin{aligned}
& \frac{\partial \delta}{\partial t}+\frac{1}{a} \nabla \cdot \boldsymbol{v}+\frac{1}{a} \nabla \cdot(\delta \boldsymbol{v})=0, \\
& \frac{\partial \boldsymbol{v}}{\partial t}+\frac{\dot{a}}{a} \boldsymbol{v}+\frac{1}{a}(\boldsymbol{v} \cdot \nabla) \boldsymbol{v}=-\frac{1}{a} \nabla \psi-\frac{1}{4 \pi a \rho}\left(\boldsymbol{B}_{\mathbf{0}} \times \nabla \times \boldsymbol{b}\right. \\
& +\boldsymbol{b} \times \nabla \times \boldsymbol{b}), \\
& \frac{1}{a^{2}} \Delta \psi=4 \pi G\left(\rho-\rho_{0}\right), \\
& \frac{\partial \boldsymbol{B}}{\partial t}+2 \frac{\dot{a}}{a} \boldsymbol{B}=\frac{1}{a} \nabla \times(\boldsymbol{v} \times B),
\end{aligned}
$$

where $\delta(t, r)=\left(\rho-\rho_{0}\right) / \rho_{0}(t)$ is the matter density contrast and $\boldsymbol{b}(t, r)$ is the magnetic field perturbation i.e. $\boldsymbol{B}(t, r)=\boldsymbol{B}_{0}(t)+$ $\boldsymbol{b}(t, r)^{1}$. In the epoch when the condition of low energy density associated with the magnetic field is valid i.e. $B^{2} / \rho \ll 1$, the evolutionary perturbation scheme may be simplified. The last expression in the Euler equation consists of two terms; the first may be ignored due to the small value of $\boldsymbol{B}_{0}$ and the second is proportional to $\nabla \rho \propto \nabla|\boldsymbol{b}|$, as can be seen from Eqs. (11) and (20) below. We shall assume in the first approximation that

\footnotetext{
${ }^{1}$ For simplicity, it is assumed here that the background field is represented by its uniform component. Following Barrow et al. (1997), its sufficiently small initial value (i.e. $B_{0}<10^{-9} \mathrm{G}$ ) does not affect either the background dynamics or the CMB spectrum. The obtained final results on $B$ distribution are not expected to depend on this (unrealistic on a few Mpc scale) assumption.
}

it is also small compared to the density potential gradient. Thus in the first iteration step we postulate a force-free magnetic field. The Faraday equation describes the magnetic field scaling as a function of accretion velocity. Both the amplified magnetic field and the density compression are coupled with the velocity field but the induction equation decouples from the hydrodynamic equations. Therefore its formal solution may be given independently. Applying the assumed symmetries and transforming the r.h.s. of Eq. (9) one obtains the induction equation in the integrable form.

$\frac{\mathrm{d} \boldsymbol{B} a^{2}}{\mathrm{~d} t} \equiv \frac{\partial \boldsymbol{B} a^{2}}{\partial t}+\left(\frac{\boldsymbol{v}}{a} \cdot \nabla\right)\left(\boldsymbol{B} a^{2}\right)=-\left(\boldsymbol{B} a^{2}\right) \nabla \cdot\left(\frac{\boldsymbol{v}}{a}\right)$.

Its formal solution is

$\boldsymbol{B} a^{2}=\boldsymbol{B}_{0} \exp \left[-\int \nabla \cdot\left(\frac{\boldsymbol{v}}{a}\right) \mathrm{d} t\right]$,

where $\boldsymbol{v}$ as well as $\rho$ and $\psi$ are given by the solutions of Eqs. (6)-(8). The successive iterative procedure will couple in the next steps the Faraday and gravitational perturbations equations, including the magnetic force to the r.h.s. of Euler equation. However this is not essential before $|\boldsymbol{B}| \approx \psi$.

\section{Nonlinear cylindrical perturbation}

The dynamics of collapsing structure modeled from a pressureless fluid is governed by Eqs. (6)-(8). Applying the assumed form of accretion velocity, the dynamic equations for the fluid transform the continuity equation into

$\frac{\partial \delta}{\partial t}+\frac{1}{a r} \frac{\partial r v_{r}}{\partial r}+\frac{1}{a}\left(\frac{\delta}{r} \frac{\partial\left(r v_{r}\right)}{\partial r}+v_{r} \frac{\partial \delta}{\partial r}\right)=0$,

two components of the Euler equation become

$$
\begin{aligned}
& \frac{\partial v_{r}}{\partial t}+\frac{\dot{a}}{a} v_{r}+\frac{1}{a}\left(\frac{v_{r}}{r} \frac{\partial r v_{r}}{\partial r}-\frac{1}{r^{3}}\left(r^{2} v_{r}^{2}+r^{2} v_{\theta}^{2}\right)\right)=-\frac{1}{a} \frac{\partial \psi}{\partial r} \\
& \frac{\partial v_{\theta}}{\partial t}+\frac{\dot{a}}{a} v_{\theta}+\frac{1}{a} \frac{v_{r}}{r} \frac{\partial r v_{\theta}}{\partial r}=0
\end{aligned}
$$

The peculiar gravitational potential satisfies the Poisson equation

$$
\frac{1}{r a^{2}} \frac{\partial}{\partial r}\left(r \frac{\partial \psi}{\partial r}\right)=\alpha \delta
$$

where $\alpha(t) \equiv 4 \pi G \rho_{0}$. 
Many authors (see for example, Lifshitz 1946; Groth \& Peebles 1975; Peebles 1980) have studied the analytical solutions for the linearized problem of perturbation evolution in an expanding Universe. In several cases the nonlinear solutions have also been developed (Zeldovich 1970). Yet, in general, the high degree of complexity of the nonlinear set of partial differential equations does not allow one to obtain analytical solutions. In this paper we also attempt to obtain the particular nonlinear solution for growing structure. Obviously, to proceed, additional assumptions are necessary. The problem becomes much simplified when we adopt that the tangential velocity component is small relative to the radial one i.e. $\left(v_{\theta} / v_{r}\right)^{2} \ll 1$. This results in a separation of Eq. (14). The above assumption is consistent with the small value of the initial vorticity and the resulting equations reduce to the equation set (12)-(13) and (15), describing the rotationless flow of the magnetized fluid.

To obtain a convenient form, we choose first the new time coordinate $x=a^{-1 / 4}=t^{-1 / 6}$, instead of the cosmic time $t$. Our analysis becomes more clear when we use the rescaled variables. Replacing the old functions $\delta, v_{r}, \psi$ by the new variables $X, z_{2}, z_{4}$, where $X=\delta, z_{2}=a r v_{r}$ and $z_{4}=r^{3} a^{2} \frac{\partial \psi}{\partial r}$ and defining the new independent variable $\xi \equiv r / x$, the continuity, the Euler and Poisson equations read

$z_{2}^{\prime}(\xi)(1+X(\xi))+\left(\frac{1}{6} \xi^{2}+z_{2}(\xi)\right) X^{\prime}(\xi)=0$,

$-z_{2}^{2}(\xi)+\xi\left(\frac{1}{6} \xi^{2}+z_{2}(\xi)\right) z_{2}^{\prime}(\xi)+z_{4}(\xi)=0$,

$-\beta \xi^{4} X(\xi)-2 z_{4}(\xi)+\xi z_{4}^{\prime}(\xi)=0$,

where prime means the differentiation with respect to $\xi$ and $\beta \equiv 4 \pi G\left(\rho_{0} a^{3}\right)$ (hereafter we take its value as $\beta=\frac{2}{3}$ ). This scaling is not only a mathematical manipulation. Introducing the $\xi$-variable reduces the differential complexity of the problem, allowing us to substitute the partial by the ordinary differential equations. In effect, the Eqs. (16)-(18) govern the self-similar behaviour of the fluid motion, leading to the nonlinear 3rd-order equation for the rescaled velocity $z_{2}(\xi)$

$$
\begin{gathered}
\xi^{3}\left[\frac{1}{6} \xi^{2}+z_{2}(\xi)\right]^{2} z_{2}^{(3)}(\xi)+\xi^{2}\left[\frac{1}{6} \xi^{2}+z_{2}(\xi)\right] \\
\cdot\left[-5 z_{2}(\xi)+\xi\left(\frac{1}{6} \xi+4 z_{2}^{\prime}(\xi)\right)\right] z_{2}^{\prime \prime}(\xi) \\
-\xi\left[-15 z_{2}^{2}(\xi)+4 \xi z_{2}(\xi)\left(-\frac{1}{2} \xi+2 z_{2}^{\prime}(\xi)\right)\right. \\
\left.+\xi^{2}\left(\frac{25}{36} \xi^{2}+\frac{2}{3} \xi z_{2}^{\prime}(\xi)-z_{2}^{\prime 2}(\xi)\right)\right] z_{2}^{\prime}(\xi) \\
-8\left[\frac{1}{6} \xi^{2}+z_{2}(\xi)\right] z_{2}^{2}(\xi)=0 .
\end{gathered}
$$

The detailed analysis of the above dynamical set (16)-(18) may be performed either through the qualitative methods of its asymptotic form or requires numerical integration.

Nevertheless, some general relation may be derived even before the solutions are obtained. The continuity Eq. (16) implies the generalized expression for density contrast and velocity function $z_{2}$.

$\ln (1+X)=-\int \frac{z_{2}^{\prime}(\xi)}{\frac{1}{6} \xi^{2}+z_{2}(\xi)} \mathrm{d} \xi=-\int \frac{\nabla \cdot \boldsymbol{v}}{a} \mathrm{~d} t$

This is a nonlinear counterpart of the known linear relation i.e. $\operatorname{div} \boldsymbol{v} \propto X$ (e.g., Willick \& Strauss 1998), coupling $\rho$ vs. $v$ and thus $B$ vs. $v$. Lacking the analytical solutions we note that the asymptotic form for large velocities $\left|z_{2}\right| \gg \xi^{2}$ predicts the asymptotic property of the density contrast $X+1 \cong\left|z_{2}\right|^{-1}$.

The linearization of the Eqs. (16)-(18) and its resulting solution for $z_{2}$ allows us to compare the time dependent part of $v_{r}$ with the linear regime expressions $v_{1,2} \propto a^{1 / 2}, a^{-2}$ (Peebles 1980). Finally, one has

$\left(z_{2}^{\operatorname{lin}}\right)_{1}=-\xi^{6}, \quad\left(z_{2}^{\operatorname{lin}}\right)_{2}=-\xi^{-4}$

and the implied linear solutions for density contrast are

$\left(X^{\operatorname{lin}}\right)_{1}=9 \xi^{4}, \quad\left(X^{\operatorname{lin}}\right)_{2}=4 \xi^{-6}$.

The analysis of the asymptotic behaviour of Eqs. (16)-(18) at infinity $(\xi \rightarrow \infty)$ becomes straightforward when we define the new variable $\zeta, \xi \rightarrow 1 / \zeta$. The subsequent linearization of the above equation set gives for $\xi \rightarrow \infty$ the following solutions for the velocity measure

$\left(z_{2}^{\operatorname{lin}}\right)_{1}=-\zeta^{-6},\left(z_{2}^{\operatorname{lin}}\right)_{2}=-\zeta^{4}, \quad\left(z_{2}^{\operatorname{lin}}\right)_{3}=$ const.

and the density contrast

$\left(X^{\operatorname{lin}}\right)_{1}=9 \zeta^{-4}, \quad\left(X^{\operatorname{lin}}\right)_{2}=4 \zeta^{6}$.

The above enables us to state that both velocity field and density contrast have solutions vanishing for $\xi \rightarrow \infty$. Below we deal with the physical solutions i.e. regular at $\xi=0$ and at $\xi \rightarrow \infty$.

\section{Linear and nonlinear magnetic amplification}

The adiabatic matter compression determines the field amplification through the reached value of $\rho$. The amplification degree is controlled by the compression symmetry (see Eq. (10)). For cylindrically collapsing structures one obtains the magnetic field amplification $W_{B} \equiv \frac{B}{B_{0}} a^{2}$, thus taking into account Eqs. (11) and (20) one has $\frac{W_{B}}{1+X}=$ const., while for example, in the case of spherical symmetry this quantity would be given by $\frac{W_{B}}{(1+X)^{2 / 3}}=$ const. On the other hand, the frozen field amplification expressed by the solution of the induction equation is defined by the velocity field given by Eq. (19). To represent it directly by the time evolution of the velocity field we first present the velocity divergence in old variables $(x, r)$

$\nabla \cdot \boldsymbol{v}=\frac{1}{r} \frac{\partial\left(r v_{r}\right)}{\partial r}=\xi^{-1} x^{2} \frac{\mathrm{d} z_{2}}{\mathrm{~d} \xi}=-\frac{x^{5}}{r^{2}} \frac{\partial z_{2}}{\partial x}$.

Note that the "self-similar" variable $\xi$ is used here only for differentiation, since we have $x \frac{\partial}{\partial r}=\frac{\mathrm{d}}{\mathrm{d} \xi}=-\frac{x}{\xi} \frac{\partial}{\partial x}$. The exponent in Eq. (11) becomes

$\int \frac{\nabla \cdot \boldsymbol{v}}{a} \mathrm{~d} t=\frac{1}{r^{2}} \int x^{2} \frac{\partial z_{2}}{\partial x} \mathrm{~d} x$. 
Replacing, in the above, the time variable by the scale factor $a$ and assuming the separability of the velocity function $v_{r}=-V(r) v(a)$ (for collapse we have $v_{r}(r, t)<0$ ), one obtains the alternative form of Eq. (26).

$-\int \frac{\nabla \cdot \boldsymbol{v}}{a} \mathrm{~d} t=\frac{V(r)}{r} \int\left(a^{\frac{1}{2}} \frac{\partial v(a)}{\partial a}+v(a) a^{-\frac{1}{2}}\right) \mathrm{d} a$.

Thus the final expression for density and magnetic field compression due to nonlinear structure formation reads

$W_{B}=X+1=\exp \left[\frac{V(r)}{r} \int\left[a^{\frac{1}{2}} \frac{\partial v(a)}{\partial a}+v(a) a^{-\frac{1}{2}}\right] \mathrm{d} a\right]$.

Combining the above equation, for the growing mode $(v(a) \propto$ $a^{1 / 2}$ ) of the velocity field and Eq. (21) yields the following amplification rate in the linear regime

$W_{B}=\exp \left[\frac{3 V(r) a}{2 r}\right]=\exp \left[\frac{3}{2} r^{4} a\right]=\exp \left[\frac{3}{2} \xi^{4}\right]$.

The successive discussion of nonlinear behaviour will also deal with the growing mode. Knowledge of the exact nonlinear amplification $W_{B}=\exp \left[-\int \nabla \cdot\left(\frac{v}{a}\right) \mathrm{d} t\right]=\exp \left[-\int \frac{z_{2}^{\prime}(\xi)}{\frac{1}{6} \xi^{2}+z_{2}(\xi)} \mathrm{d} \xi\right]$ needs the solution of the very complex, quasilinear Eq. (19) for $z_{2}$. The zero value of the coefficient at the $z_{2}^{(3)}$ term allows us to determine the singularity points of this equation i.e. $\xi=0$ and $\frac{1}{6} \xi^{2}+z_{2}=0$. Since we postulate the regular velocity solution at the structure centre we focus here on a later one, which appears when the overdensity grows in the nonlinear regime. The singularity point position depends on the chosen boundary condition (a movable singularity). This is specific to the nonlinear differential equations (Bender \& Orszag 1978). Physically, the emergence of a singularity in the velocity and density field is an artefact of the collisionless fluid. This feature has also been found in $N$-body simulations and the test-particle approach (e.g., Bertschinger 1985) to gravitational collapse, as an outer caustic which forms around the structure. (For collisional matter, $p \neq 0$, it becomes a shock surface.)

Since its analytical solution is difficult to obtain, below we present the results of numerical integration below the caustic surface. Independently we verify the asymptotic behaviour of the solution at $\xi \rightarrow \infty$. As expected from Eq. (23), the velocity function $z_{2}(\xi)$ and the density contrast $X(\xi)$ approach zero at infinity. The principal features of the particular solution inside the structure and its asymptotic behaviour at infinity are illustrated in Figs. 1 and 2 below. These figures show the density contrast and the velocity field as a function of $\xi$ (or $\zeta$ ) in the log-log scale. Both quantities are expressed respectively, in units of $\left(X^{\operatorname{lin}}\right)_{1}$ and $\left(z_{2}^{\operatorname{lin}}\right)_{1}$ or in units of $\left(X^{\operatorname{lin}}\right)_{2}$ and $\left(z_{2}^{\operatorname{lin}}\right)_{2}$ at infinity. The presented profiles are only qualitative, in the sense that they are given by the numerical integration for a required set of the particular boundary conditions. The boundary conditions for numerical integration were taken from the linear solutions of Eqs. (16)-(18). They clearly display two distinctive regions corresponding to linear and nonlinear formation rates. Roughly, the linearity is represented by the straight line $\frac{X}{\left(X^{\text {lin }}\right)_{1}} \cong 1$ and the nonlinear region of the magnetic field and density contrast amplification for $\xi \geq 0.2$ shows a strongly
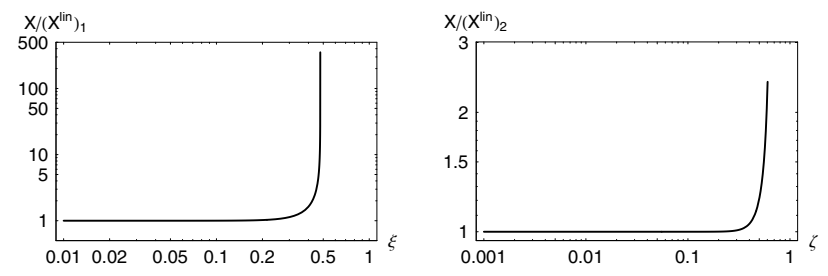

Fig. 1. The nonlinear excess of the density contrast $X / X^{\text {lin }}$ below the discontinuity (left panel), as a function of the $\xi$-variable and (right panel), above the discontinuity as a function of the $\zeta$-variable.
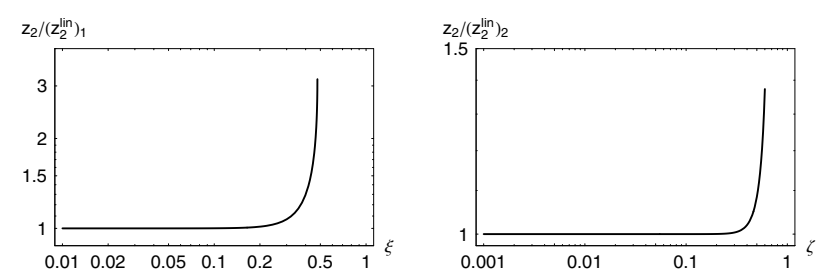

Fig. 2. The nonlinear excess of the velocity measure $z_{2} / z_{2}^{\text {lin }}$ below the discontinuity (left panel), as a function of the $\xi$-variable and (right panel), above the discontinuity as a function of the $\zeta$-variable.

growing curve. It should be noted that the validity of the linear approach is slightly different in the case of density contrast and the velocity function ${ }^{2}$.

The direct comparison of the linear and nonlinear amplification rate for a given structure radius shows that the nonlinear compression implies the magnetic field growth rate of several orders of magnitude. The predicted (cf. Fig. 1) amplification of the order of $W_{B} \sim 10^{3}$ within the range $\left(\xi_{1}, \xi_{2}\right)=$ $(0.28,0.48)$ corresponds to the evolutionary period given by $\frac{a_{2}}{a_{1}}=\left(\frac{\xi_{2}}{\xi 1}\right)^{4} \approx 6.5$. Thus, the magnetic growth of the order of $2 \times 10^{3}$ may be attributed to the early nonlinear structure evolution between, for instance, redshift $\sim 6$ and redshift $\sim 0$. Certainly, the magnetic amplification becomes much greater as nonlinear evolution proceeds but then the validity of the applied assumptions becomes questionable.

\section{Conclusions}

The goal of this paper is to present the amplification rate of the magnetic field associated with the forming gravitational structure of cylindrical symmetry. The widespread conviction that the large-scale structures are filled with microgauss cosmological magnetic fields motivates our interest in amplification processes during their evolution. On the other hand, we see a high degree of filamentarity in the galaxy redshift surveys (e.g., Sathyaprakash et al. 1998). This demonstrates that we deal with magnetized, elongated structures of axial symmetry.

To determine their magnetic structure growth, several simplifications are needed. We used here two categories of

\footnotetext{
2 The density growth $\frac{X}{\left(X^{\operatorname{lin})_{1}}\right.}$ is faster than the velocity growth $\frac{z_{2}}{\left(z_{2}^{\operatorname{lin}}\right)_{1}}$. It is clearly seen when the respective values are compared i.e. $\frac{X}{\left(X^{\operatorname{lin})_{1}}\right.}(0.2)=1.02, \frac{X}{\left(X^{\operatorname{lin})_{1}}\right.}(0.47)=6.15$ and $\frac{z_{2}}{\left(z_{2}^{\operatorname{lin}}\right)_{1}}(0.2)=1.01$, $\frac{z_{2}}{\left(z_{2}^{\operatorname{lin}}\right)_{1}}(0.47)=2.18$.
} 
simplifying assumptions: physical (i.e. $p \sim 0$ and $F_{L} \sim 0$ ), constraining the results to the early nonlinear phase and geometrical ones - requiring the radial motions and thus the axial fields. On the basis of a formal solution of the induction equation we obtained the exact analytical expression for linear field amplification. The relationship between the density contrast and the magnetic field strength is established through the velocity field divergence. However the major conclusion concerns the nonlinear phase. The magnetic field may be effectively enhanced there. The density contrast growth is stronger than the velocity, achieving the nonlinearity regime earlier. The radial structure of the magnetic field and density contrast are identical, in general - nonhomogeneous.

Contrary to this highly idealized model, in the realistic situation, the centrifugal forces will stop the collapse. This will however occur in the successive, virialization phase, when the matter will become collisional and then shocked. Therefore, a proper description requires more elaborate application of the fluid model. Within its current limitations the above applied symmetries seem to be less weighty than the physical assumptions. According to previous papers (e.g., Siemieniec \& Woszczyna 2004; Bruni et al. 2003) the more degenerate, pancake geometry leads to comparable amplification results. Introducing cylindrical symmetry enables us instead to depict the magnetic field profile inside the structure. The substantial enhancement of the matter density accreting onto collapsing structure indicates that significant magnetic fields may be produced in its outer region - the precursor of the future shock.
Acknowledgements. We thank the referee, Dr. G. Sigl for criticisms and useful comments. This work was supported in part by "Komitet Badań Naukowych" projects: "Cosmic magnetic fields" (G.S.-O.) and "Rigorous description of scalar and tensor perturbations in flat and open Friedmann universes" (Z.A.G.).

\section{References}

Barrow, J., Ferreira, P., \& Silk, J. 1997, PRL, 78, 3610

Bender, C. M., \& Orszag, S. A. 1978, Advanced Mathematical Methods for Scientists and Engineers (New York: McGraw-Hill Book Company)

Bertschinger, E. 1985, ApJS, 58, 39

Bruni, M., Maartens, R., \& Tsagas, C. 2003, MNRAS, 338, 785

Groth, E., \& Peebles, P. 1975, A\&A, 41, 143

Kim, E., Olinto, A., \& Rosner, R. 1996, ApJ, 468, 28

Lesch, H., \& Chiba, M. 1995, A\&A, 297, 305

Lifshitz, E. 1946, J. Phys. (USSR), 10, 116

Peebles, P. 1980, The Large-Scale Structure of the Universe (Princeton: Princeton Univ. Press)

Peebles, P. 1993, Principles of Physical Cosmology (Princeton: Princeton Univ. Press)

Sathyaprakash, B., Sahni, V., \& Shandarin, S. 1998, ApJ, 508, 551

Siemieniec, G., \& Woszczyna, A. 2004, A\&A, 414, 1

Wasserman, I. 1978, ApJ, 224, 337

Willick, J., \& Strauss, M. 1998, ApJ, 507, 64

Zeldovich, Y. 1970, A\&A, 5, 84

Zeldovich, Y., Ruzmaikin, A., \& Sokolov, D. 1980, Magnetic Fields in Astrophysics (NY: McGraw-Hill) 